\title{
Wolter Optics for Neutron Focusing
}

\author{
D.F.R. Mildner ${ }^{1}$ and M.V. Gubarev ${ }^{2}$ \\ ${ }^{1}$ National Institute of Standards and Technology, Gaithersburg, MD 20899 \\ ${ }^{2}$ Marshall Space Flight Center, NASA, Huntsville, AL 35812
}

\begin{abstract}
Focusing optics based on Wolter optical geometries developed for x-ray grazing incidence beams can be designed for neutron beams. Wolter optics are formed by grazing incidence reflections from two concentric conic sections (for example, a paraboloid and a hyperboloid). This has transformed observational X-ray astronomy by increasing the sensitivity by many orders of magnitude for research in astrophysics and cosmology. To increase the collection area, many reflecting mirrors of different diameters are nested with a common focal plane. These mirrors are fabricated using nickel-electroformed replication techniques. We apply these ideas to neutron focusing using nickel mirrors. We show an initial test of a conical mirror using a beam of cold neutrons.
\end{abstract}

key words: electroformed nickel replication, focusing optics, grazing angle incidence, mirror reflection, neutron focusing, Wolter optics

\section{Introduction}

Available neutron sources have a lower brilliance by many orders of magnitude than $\mathrm{x}$ ray sources that are conveniently provided by synchrotron radiation. This means that optical phenomena are generally not as easy to observe with neutrons as at conventional light and $\mathrm{x}$-ray sources. Consequently, techniques that can focus and concentrate neutron beams with suitable optics are important for making higher neutron current densities available for experiments. This is particularly attractive when the neutron method is the most appropriate for the measurement. Many of the techniques for focusing neutrons have been adapted from devices that have been developed for focusing X-rays. These include techniques that use diffraction (such as Fresnel lenses and pinholes), refraction (such as biconcave lenses [1]) or reflection (such a neutron mirrors using grazing incidence for total reflection and multilayers). In addition, neutrons can be focused by refraction in an inhomogeneous magnetic field provided by a magnetic hexapole [2]. We now consider optics involving grazing angle incidence that have revolutionized observational X-ray astronomy. We note that neutron sources are extended, and therefore they neither produce parallel beams nor may be considered as point sources. 


\section{Grazing Angle Incidence}

Successful neutron optical devices are now commonplace in instrumentation for scientific research, particularly for use with cold or long-wavelength neutrons. This is because the optical properties of materials for both $\mathrm{X}$-rays and neutrons have refractive indices less than unity. The scattering amplitude of the nucleus is given by $b=b^{\prime}-i b^{\prime \prime}$, where the real part $b^{\prime}$ is the coherent scattering length and the imaginary part $b^{\prime \prime}$ is responsible for the absorption of neutrons. The neutron optical properties of a material are determined [3] by its refractive index given by $n=1-\delta+i \beta=1-\lambda^{2} \rho b^{\prime} / 2 \pi+i \lambda \rho \sigma_{a} / 4 \pi$, where $\lambda$ is the neutron wavelength, $\rho$ is the average atomic density of the material, and $\sigma_{a}=2 \lambda b^{\prime \prime}$ is the absorption cross section. The value of $\delta$ depends on the ratio of the interaction of the neutron with the medium and the neutron energy. The refractive index for most materials differs from unity by a few parts in $10^{5}$ for thermal neutrons with wavelengths $\lambda>1 \AA$,. With few notable exceptions, most materials have absorption cross sections that are almost zero, so that $n=1-\delta$ (and we write $b^{\prime}=b$ ). Consequently, thermal and cold neutrons may be reflected at grazing incidence angles by total external reflection, or reflected at the boundaries of different materials, in a similar fashion for X-rays. The advantage of reflective optics is that they are achromatic compared to refractive optics.

Kirkpatrick and Baez [4] originally developed grazing angle incidence for $x$-ray focusing using one-dimensional parabolic mirrors. This has now been adapted for focusing with neutrons [5]. The difficulty with grazing incidence optics is that a near parallel beam incidence is required and so that the optic must be placed far enough from the source to ensure small incident beam divergence. The optic focuses in one plane, so that a second mirror mounted orthogonally to the first can achieve two-dimensional focusing. K-B neutron mirrors using two successive reflections in orthogonal directions can efficiently focus neutron beams into a small area with an incident divergence that is limited by the mirror critical angle. The size of the focal spot is primarily determined by geometrical demagnification of the source and by figure errors in the mirror shape. Approximately two orders of magnitude in neutron current density increase can be achieved within a spot of dimension $\sim 100 \mu \mathrm{m}$ using crossed mirrors. At present K-B mirror focusing is limited to neutron beams with small cross sections; such an optic that focuses onto the sample has been recently incorporated into a high-pressure diffractometer on the SNS pulsed neutron source [6]. Furthermore, parabolic K-B mirrors can be nested to increase the effective collection area. Similarly, capillary optics has been developed for focusing Xrays, and the technique has also been applied to neutrons [7]. Unfortunately the focal lengths are too short for most applications except for measurements that involve neutron absorption, such as prompt gamma activation analysis and neutron depth profiling.

One of the difficulties in applying x-ray focusing techniques to neutron beams is the size of the source. X-rays from a synchrotron may be considered as point sources, and the advantage of an ellipsoidal mirror is that all the trajectories emanating from one focus (the source) reach the other focus. Similarly, rays from a parallel beam, from an astronomical source for instance, incident along the axis of a parabolic mirror are reflected to the focus. On the other hand, neutron sources are extended, as well as being less brilliant compared to $\mathrm{x}$-ray sources. Consequently most trajectories within a neutron 
beam are non-axial. This is true even for a quasi-parallel beam formed by a collimation system since their cross sections must be relatively large in order to preserve a reasonable flux. Consequently terrestrial neutrons sources cannot be considered as points; nor can they be considered as producing parallel beams. Hence most trajectories emanate from off-axis points, resulting in higher orders of aberrations compared to the on-axis point source. Though focusing systems based on reflective optics do not suffer from the chromatic aberrations of refractive optics, a further complication arises from the fact that the neutron has mass. For a beam with finite wavelength resolution (and hence velocity spread) there will be gravitationally chromaticity, though this effect is less than chromatic aberrations from refractive optics.

Various efforts have been made to use curved mirrors to focus neutron beams using single reflection systems, such as ellipsoidal mirrors for point-to-point focusing. However the principal disadvantage of single reflection mirror systems is the aberration caused by off-axis trajectories. This produces coma resulting from differences in the angular magnification for reflections from different areas of the mirror located along the same mirror meridian. This image deterioration increases with the mirror length, and hence the angular range for a single reflection system is limited. The aberration from coma is always present in one-reflection systems and cannot be corrected. Furthermore, relaxing the figure requirements for ellipsoidal or parabolic mirrors results in both spherical aberration and astigmatism. The use of a toroidal mirror, however, may eliminate astigmatism that results from the difference in meridional and sagittal focal distances. This concept has been incorporated into the USANS spectrometer KW3 at the FRM-II reactor in Germany [8].

\section{Wolter optics}

Wolter [9] has proposed optics based on reflections from two surfaces in order to overcome the disadvantage of the aberration caused by off-axis trajectories for single reflection mirror systems. Grazing angle reflective optics based on two-bounce Wolter geometries are used extensively in x-ray astronomy because they minimize the aberrations. The scheme consists of grazing incidence reflections from two concentric conic sections. They take advantage of the properties of three mathematical curves (see Figure 1):

1. ellipsoid - all rays emanating from one focus converge onto the other focus

2. paraboloid - all incoming rays of a plane wave parallel to the axis are reflected to the focus

3. hyperboloid - all rays emanating from one focus are reflected as though they originated from the other focus.

In Wolter type I optics, such as a paraboloid-hyperboloid mirror shown in Figure 2, an incoming parallel beam is first reflected from the inner surface of the paraboloid such that they are reflected towards its focus. If that focus is also placed at the focus of the hyperboloid, the rays are further reflected from the inner surface of the hyperboloid to its other focus. Note for completion that in Wolter type II optics, an incoming parallel beam 
is reflected from the inner surface of the paraboloid and then the outer surface of the hyperboloid, giving a much longer focal length, but this does lend itself easily to nested surfaces. Finally, Wolter type III optics involves reflections of an incoming parallel beam from the outer surface of a paraboloid, followed by reflection from the inner surface of the ellipsoid. In practice, we consider only Wolter type I optics.

The advantage of the Wolter optics is that the necessary angular displacement for focusing is achieved by two half-angle reflections compared to the full angular displacement of K-B mirrors. In addition, these confocal surfaces of revolution eliminate on-axis coma, and reduce off-axis coma. Furthermore, since all reflections are at grazing angles this allows mirrors of different sizes to be nested within each other, such that they have a common focus. This enables a great increase in the collection area of the optic.

Figure 2 shows the geometry of a Wolter type I optics formed by grazing-incidence reflections from two concentric conic sections, a paraboloid and a hyperboloid. Parallel rays reflect from the parabolic section and then the hyperbolic section, forming an image at the focus. All reflecting mirrors of different diameters have the same focal length $f$, which is defined as the distance from the intersection of the paraboloid and the hyperboloid to the focal point. If the intersection of a given cone is at a radius $r$ from the axis, then the mean grazing angle $\alpha$ at the paraboloid surface for rays parallel to the optic axis is given by $\tan 4 \alpha=(r / f)$. Aberrations resulting from off-axis rays may be minimized when the grazing angle at both the parabolic and the hyperbolic surfaces are equal. This is the case when the parabolic surface is at an angle $\alpha$ to the optic axis and the hyperbolic surface is at an angle of $3 \alpha$ to the axis. This geometry results in high resolution and good off-axis performance. Such devices are used in X-ray astronomy such as the Chandra X-ray Observatory [10].

\section{Electroformed Nickel Replication}

Figure 3 shows an x-ray optic module used for x-ray observations. Suitable cones are fabricated by a replication technique whereby a perfect copy is made of a smooth surface that has the required figure. The production of the original masters or mandrels is time consuming, since the outer surface must be made smooth to the correct figure and the required tolerances by grinding and polishing. The advantages are that once the mandrel is made the production of a requisite thin mirror is less laborious, that more than one mirror can be made from a given mandrel, and that much thinner mirrors can be produced than by other techniques. This replication process is suitable for the production of grazing incidence optics. The parabolic/hyperbolic surfaces are machined on the outer surface of the mandrel that subsequently highly polished. The usual material for the mandrel is nickel, and an electroformed nickel is placed on the mandrel surface to produce mirrors suitable for neutrons. These mirrors are thin and light weight and have a high reflectivity. Cryogenic cooling is used to separate the mirror from the mandrel.

The mirror surfaces must be quite smooth because diffractive scattering off surface irregularities result in a loss in intensity. If the surface irregularities are large, the specular 
reflection from these errors in the surface profile will dominate. This is particularly true for a two-reflection mirror system. To estimate the performance of an optic, we need to know the distribution of the deviations of the surface imperfections on all spatial scales of the optic, from the roughness on submicron scales to the figure errors of the optic. For most X-ray astronomy applications, this microroughness must be much less than $5 \AA$ (rms), and the tolerances of the slope errors and shape deviations are very tight. This makes the fabrication of the optics expensive. Each mirror should follow the figure of the curve as close as possible with a minimum of surface roughness. Consider an incoming neutron trajectory at an incidence angle $\alpha$ on to a mirror surface that is off by an amount $\delta r$ from its correct height $r$. The optical path difference is given by $2 \delta r \sin \alpha$, with a corresponding phase difference $\Delta=(2 \pi / \lambda) 2 \delta r \sin \alpha$. If we assume a uniformly rough mirror surface with a Gaussian height distribution, the loss in reflected intensity may be given by $\exp \left(-\Delta_{r m s}^{2}\right)$, where $\Delta_{r m s}$ is its rms value. Ideally $\Delta_{m s}$ must be very much smaller than unity. For a given wavelength and surface roughness, this loss in intensity increases with the grazing angle. For an incidence angle $\alpha=0.5^{\circ}$ and a wavelength $\lambda=4$ $\AA$, an rms roughness of $4 \AA$ will result in a $1 \%$ loss in reflected intensity. (Note that an rms roughness of $8 \AA$ results in a 5\% loss in reflected intensity.) Since the optical surface can be polished to a few $\AA$, this can be minimized for neutrons. In fact the requirements may be a little more relaxed for neutrons than for X-rays where the wavelengths are generally much shorter and the dependence on surface roughness more severe.

\section{Neutron test}

We have considered the opportunity to use an existing mirror of the Wolter I design for the focusing of thermal and cold neutrons [11]. In order to develop a useful optic, we need to take into account how we might couple a nested mirror system with a suitable detector system. This involves the type of mirror coatings, and the quality of both the surfaces and the figures. First, we must test the concept of the optic for neutrons. Fortunately, a nickel mirror produced by the electroform nickel replication process was immediately available for this initial step.

Total reflection occurs when neutrons are incident on a surface at angles below the critical angle $\theta_{c}$, given by $n=\cos \theta_{c}$, or $\theta_{c}=\lambda(\rho b / \pi)^{1 / 2}$. This defines a critical wavelength by $\lambda_{c}=(\pi / \rho b)^{1 / 2}$. Nickel has the highest critical angle per unit wavelength, $\theta_{\mathrm{c}} / \lambda=1.73 \mathrm{mrad} \AA^{-1}$ or about $0.1^{\circ} \AA^{-1}$, of all elements. Nickel $(b=10.3 \mathrm{fm})$ having a high scattering length density $\rho b=9.37 \times 10^{-6} \AA^{-2}$ with $\lambda_{c}=(\pi / \rho b)^{1 / 2}=580 \AA$ is the choice material for grazing incidence optics. Even better is isotopically pure ${ }^{58} \mathrm{Ni}(b=$ $14.4 \mathrm{fm}$ ) with a greater scattering length density of $1.31 \times 10^{-5} \AA^{-2}$, with $\lambda_{c}=490 \AA$ and $\theta_{\mathrm{c}} / \lambda=2.04 \mathrm{mrad} \AA^{-1}$. It is fortunate that nickel is already a standard coating for replicated optics for X-rays.

We have demonstrated the neutron focusing capabilities of nickel-replicated optics developed at the Marshall Space Flight Center (MSFC). We have used an existing 62 $\mathrm{mm}$ diameter cone, $175 \mathrm{~mm}$ long, with a $1 \mathrm{~m}$ focal length. The mandrel was originally 
designed as a $1 / 10$-scale version of the innermost mirror of the NASA Chandra X-ray Observatory [10]. The parabolic surface is at angle $\alpha=8.0 \mathrm{mrad}$, so that the optic has appropriate grazing angles for cold neutrons. The critical angle for total external reflection of neutrons is $1.73 \mathrm{mrad}^{-1}$ for natural nickel surface of the optic, so that the cut-off wavelength for this optic is $4.6 \AA$ for on-axis trajectories. The microroughness of the mirror is estimated to be less than $5 \AA$ from measurements of the mirror mandrel. An evaluation of the $\mathrm{x}$-ray performance of the mirror was carried out at the Stray Light Facility at MSFC. The X-ray resolution of the mirror (energy $6 \mathrm{keV}$ to $8 \mathrm{keV}$, or wavelength $1.55 \AA$ to $2 \AA$ ) defined by the half-power diameter of the mirror was found to be $0.140 \pm 0.003 \mathrm{mrad}$. This corresponds to a focal spot size of about $140 \mu \mathrm{m}$ diameter.

We have evaluated the neutron performance of this test optic using the NG-7 small-angle neutron scattering (SANS) instrument at the National Institute of Standards and Technology's Center for Neutron Research. The available neutron wavelength range of 5 $\AA$ to $20 \AA$, with a wavelength bandwidth $\Delta \lambda / \lambda=11 \%$, allows a demonstration with wavelengths close to the cut-off for the mirror. Figure 4 shows the optic mounted immediately after the beamline exit gate. Unfortunately there were a number of limitations to these test measurements caused by the parameters of the instrument. The maximum beam diameter is $25 \mathrm{~mm}$, much smaller than the diameter of the optic. This required a plate of boron nitride of thickness $13 \mathrm{~mm}$ with an annular aperture to be placed before the mirror and after the beam exit window. Consequently the incident beam illuminated only a small top portion of the mirror, corresponding to a geometric area of $17.7 \mathrm{~mm}^{2}$. The illuminated area of the mirror at the beam is only $44 \mathrm{~mm}^{2}$, compared to $272 \mathrm{~mm}^{2}$ available on the parabolic mirror.

The SANS spectrometer has a position-sensitive detector with a pixel size of $5 \mathrm{~mm}$, so that its resolution is considerably poorer than the expected focal spot size. Furthermore, the detector, which is moveable within a large vacuum tank, could not be placed as close to the optic as its focal distance. This means that a direct measurement of the focus was impossible. Instead, we took a series of measurements of the defocusing of the annular beam at different distances beyond the focus of the optic.

The optic has a graze angle of $\alpha=8 \mathrm{mrad}$ at the paraboloid, and $3 \alpha=24 \mathrm{mrad}$ at the hyperboloid. The length of the optic determines that the parabolic section presents a height of only $0.7 \mathrm{~mm}$ to the incident beam. The height of the aperture is greater than this, so that some of the incident beam misses the optic entirely, and some is incident on the hyperbolic section, which presents a height of $2.1 \mathrm{~mm}$ to the incident beam. Consequently, in addition to the straight-through beam observed on the detector, the single conical mirror may give rise to three components to the reflected beam:

(1) the intended double bounce with neutrons reflected first from the paraboloid and then from the hyperboloid, at a mean reflection angle of $4 \alpha$,

(2) a single reflection from the parabolic section that then misses striking the hyperbolic section, and is observed at a mean reflection angle of $2 \alpha$, (3) a single reflection from the hyperbolic section directly, at a mean reflection angle of $6 \alpha$, 
Component (2) arises when the incident beam is divergent or off-axis so that some fraction of the beam has an increase in the trajectory angle such that the hyperbolic section is missed. Component (3) is always present for the single conical mirror if the critical angle corresponding to the neutron wavelength is greater than the grazing angle of the hyperbolic section, that is for $\lambda>14 \AA$, although it is also present at much lower wavelengths since the incident beam is divergent. However in a nested system of mirrors this component would be absent because the parabolic section of the next inner mirror would shade the hyperbolic section. Note that component (3) comes to a focus before the true focal position of component (1).

In our test measurements the mirror was aligned with the quasi-parallel beam of the spectrometer using $10 \AA$ neutrons with all the neutron guides inserted. Though the beam at the guide exit has a divergence of $\pm 17.3 \mathrm{mrad}$, much greater than the graze angle of 8 mrad of the parabolic section, the guide exit aperture defines divergence ( $\pm 6 \mathrm{mrad}$ ) of the beam that illuminates the mirror. Figure 5 shows the image on the detector with the straight-through beam plus the three reflections. Component (3) is fainter than the other two because the tilt angle ( $24 \mathrm{mrad})$ of the hyperbolic section is greater than the critical angle of the nickel surface at $10 \AA$, and so that the intensity from this reflection is much weaker.

We have investigated the performance of the mirror by taking measurements at neutron wavelengths of $6 \AA, 10 \AA$ and $20 \AA$, and with the detector at various distances from the optic. All the neutron guides were removed, and the estimated neutron beam divergence is less than $1 \mathrm{mrad}$. Figure 6 shows the annular images taken with $20 \AA$ wavelength neutrons. Note that the component (2) is missing, since the beam divergence has been reduced considerably, so that the hyperbolic section is able to reflect all rays reflected from the parabolic section. From these results we can infer a measure of the focal spot size of $1.15 \mathrm{~mm}$ (FWHM) [11].

\section{Conclusions}

We have demonstrated the feasibility of grazing incidence optics to focus cold neutron beams using a Wolter type I optic. As a consequence we now may consider developing neutron focusing systems using such optics. These might be paraboloid-hyperboloid or paraboloid-ellipsoid configurations for quasi-parallel beams, such as for extra-terrestrial applications. Alternatively these might be hyperboloid-ellipsoid or paraboloidparaboloid for a quasi-point source. We should expect that the electroformed nickel replication process that is capable of producing X-ray optics with angular resolutions of 10 arc-seconds ( $\sim 50 \mu \mathrm{rad})$ and with graze angles less than $3.5 \mathrm{mrad}$ can also be used with thermal neutrons with similar angular resolutions. The ability of neutrons to penetrate into the bulk of most materials and also to be strongly scattered by hydrogenous materials enables neutron imaging to complement other techniques. Neutron radiography can also benefit from high resolution focusing neutron optics. 


\section{References:}

[1] M.R. Eskildsen, P.L. Gammel, E.D. Issacs, C. Detlefs, K. Mortensen, D.J. Bishop, Nature 391 (1998) 563-566.

[2] J. Suzuki, T. Oku, T. Adachi, H.M. Shimizu, TY. Hirumachi, T. Tsuchihashi, I. Watanbe, J. Appl. Cryst. 36 (2003) 795-799.

[3] V.F. Sears, Neutron Optics, Oxford University Press, 1989.

[4] P. Kirkpatrick, V. Baez, J. Opt. Soc. Am. 38 (1948) 766.

[5] G.E. Ice, C.R. Hubbard, B.C. Larson, J.W.L. Pang, J.D. Budai, S. Spooner, S.C.

Vogel, Nucl. Instrum. Meth A 539 (2005) 312-320.

[6] G.E. Ice, J.W.L. Pang, C. Tulk, J. Molaison, J.-Y. Choi, C. Vaughn, L. Lyttle, P.Z.

Takacs, K.N. Andersen, T. Bigault, A. Khounsary, J. Appl. Cryst. 42 (2009) 1004-1008.

[7] H. Chen, R.G. Downing, D.F.R. Mildner, W.M. Gibson, M.A. Kumakhov,

I.Yu. Ponomarev and M.V. Gubarev, Nature 357 (1992) 391-393.

[8] B. Alefeld, C. Hayes, F. Mezei, D. Richter, T. Springer, Physica B 234-236 (1997)

1052-1054.

[9] H. Wolter, Annalen der Physik 445 (1952) 28.

[10] http://cxc.harvard.edu/cdo/about_chandra/overview_cxo.html

[11] M.V. Gubarev, B.D. Ramsey, D.E. Engelhaupt, J.M. Burgess, D.F.R. Mildner, Nucl. Instrum. Meth B 265 (2007) 626-630 (2007).

Figure captions:

Fig 1. Schematic diagram of the three conic sections of revolution used in Wolter optics.

Fig 2. A schematic drawing of a Wolter I mirror configuration comprising a paraboloid and a hyperboloid that can focus a quasi-parallel beam to a focus.

Fig 3. An x-ray optic module with 12 nested mirrors.

Fig 4. The test mirror mounted on rotational and translation stages and installed on the NG7 beam line. At the left is the beam exit gate, and at the right is the SANS instrument box attached to the detector system. An annular aperture in the boron nitride plate placed before the optic defines the incident beam.

Fig 5. An extra-focal image taken at $4.67 \mathrm{~m}$ behind the focal point of the mirror using a

$10 \AA$ neutron beam. The image shows the straight through beam, the single reflection from the parabolic section at angle $2 \alpha$, the desired double reflection from both mirrors at $4 \alpha$, and the single reflection from the hyperbolic section at $6 \alpha$.

Fig 6. The extra-focal images of the annular aperture taken with $20 \AA$ neutrons at distances (a) $0.67 \mathrm{~m}$, (b) $2.67 \mathrm{~m}$ and (c) $4.67 \mathrm{~m}$ beyond the mirror focal spot, showing both the double reflection component (1), and the single reflection from the hyperbolic section (3). The straight-through beam is also observed. The incident beam has a sufficiently small divergence that all reflections from the parabolic section strike the hyperbolic section. 
Fig. 1

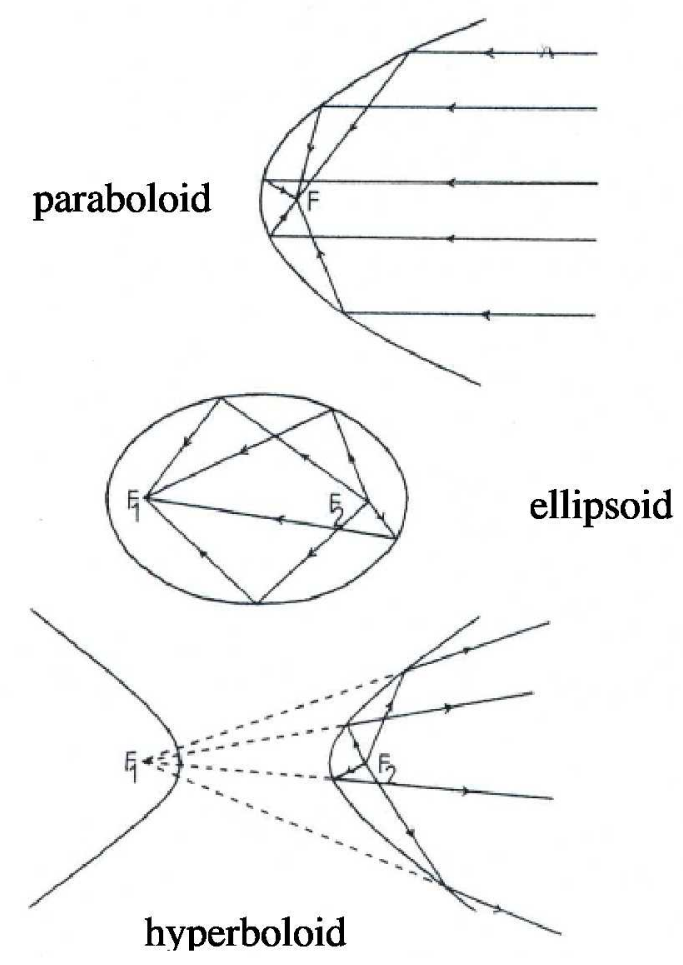

Fig. 2

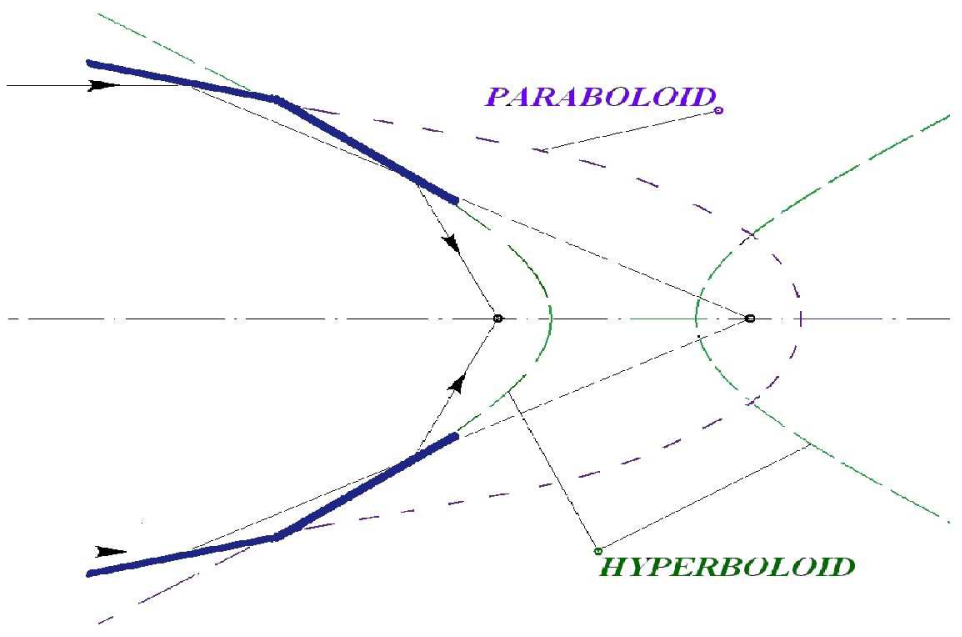


Fig. 3

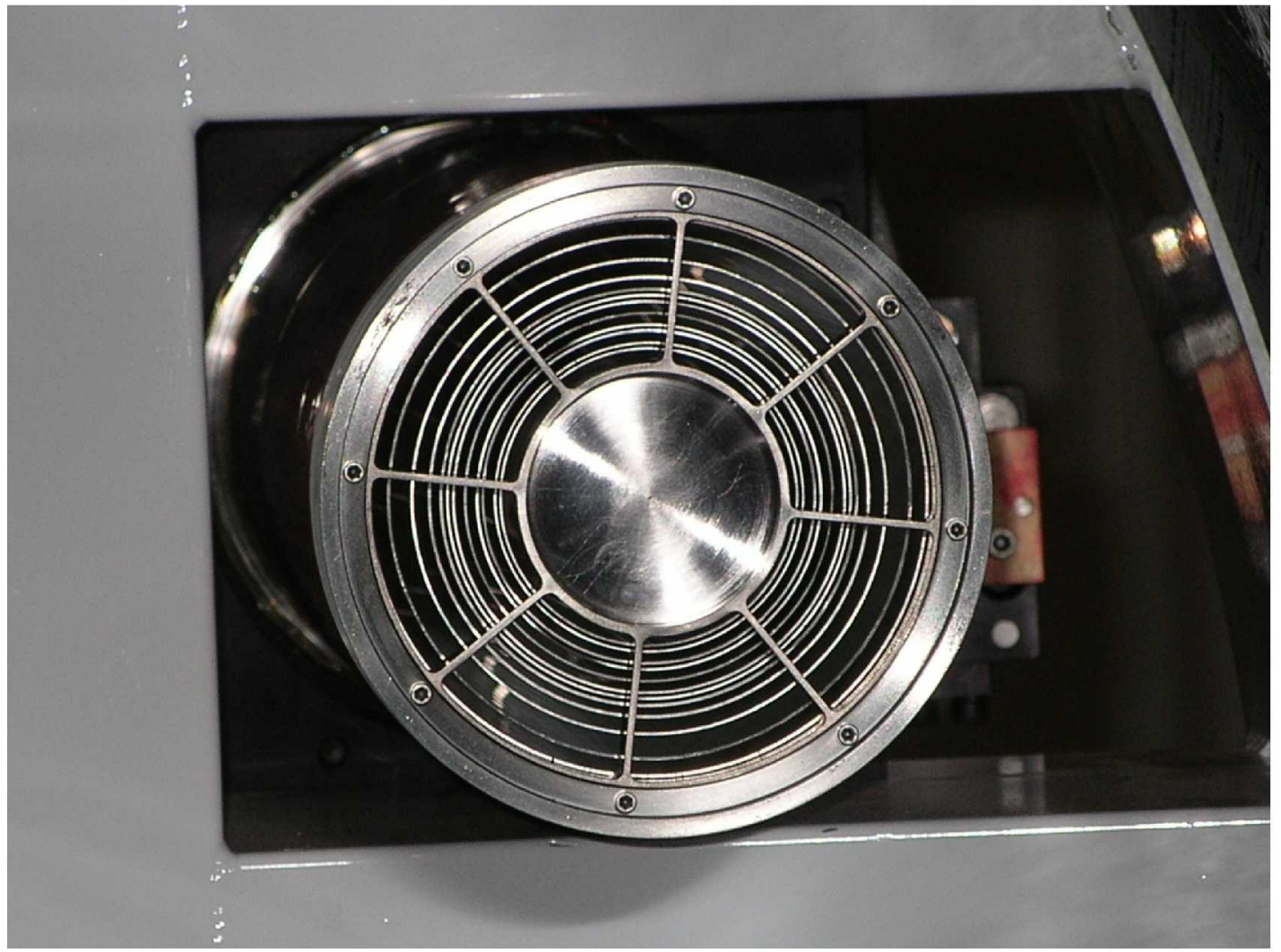

Fig. 4

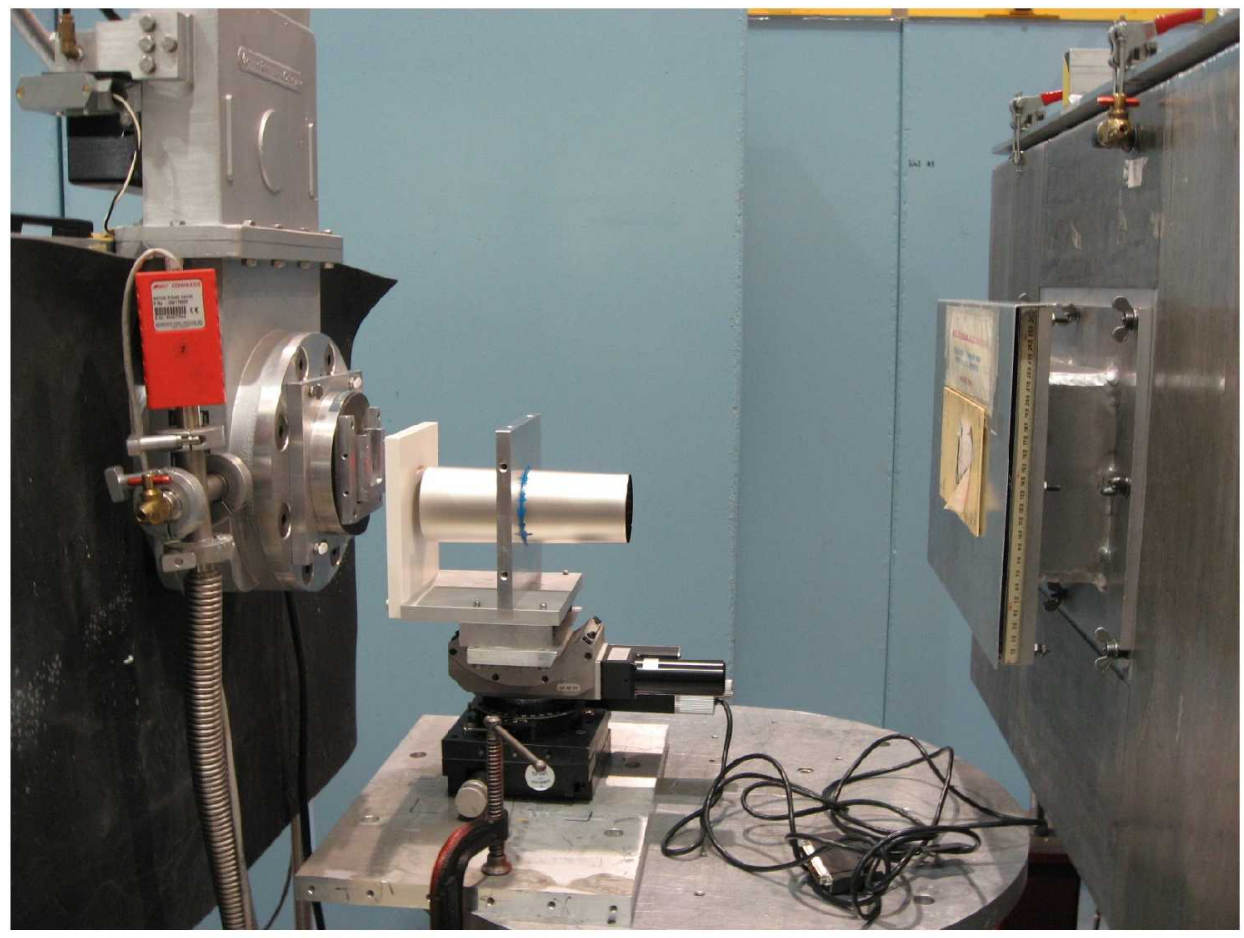


Fig. 5

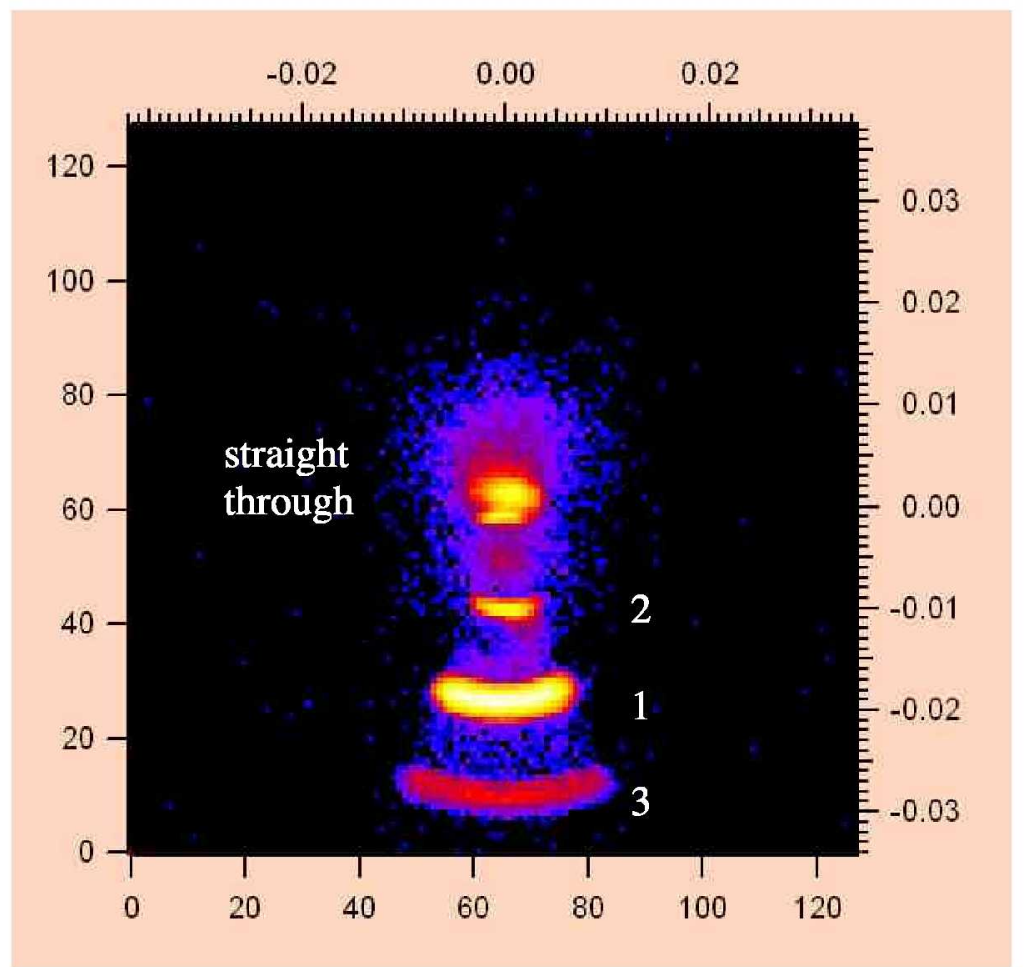

Fig. 6

(a)

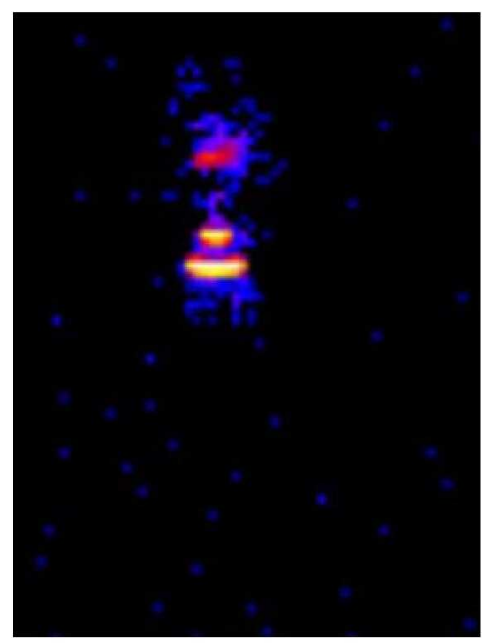

(b)

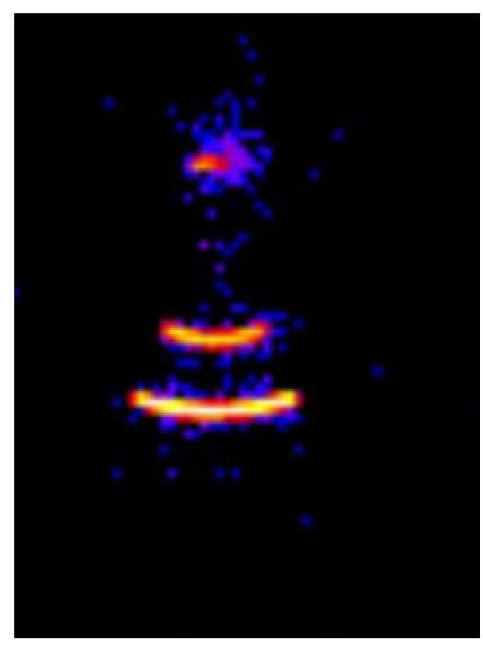

(c)

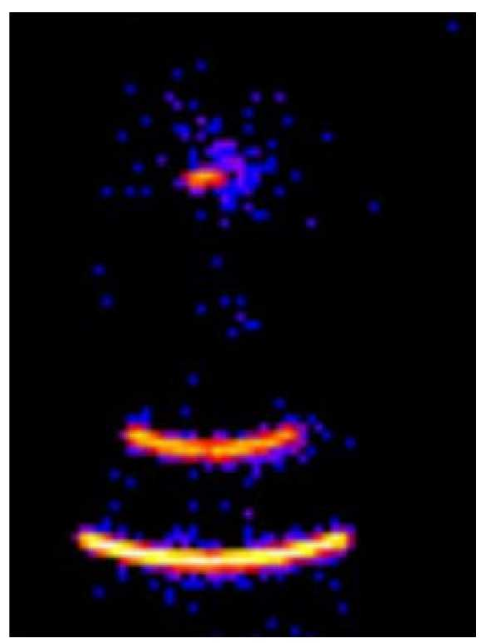

